\title{
NEW DEVELOPMENTS IN LASER ACCELERATION OF BEAMS
}

\author{
D. Umstadter, ${ }^{*}$ S. Banerjee, K. Flippo, A. Maksimchuk, K. Nemoto, N. Saleh, X. Wang, P. Zhang \\ Center for Ultrafast Optical Science, University of Michigan, Ann Arbor, MI 48109-2099 \\ V. Bychenkov, Lebedev Institute, Moscow, Russia
}

\begin{abstract}
We report experimental results in which ultra-short duration (femtosecond) laser pulses from tabletop lasers are focused to intensities above $10^{19} \mathrm{~W} / \mathrm{cm}^{2}$ onto either gas jets or thin solid-density films. At such extreme electromagnetic field strengths $\left(10^{11} \mathrm{~V} / \mathrm{cm}\right)$, plasmas are formed in which the electrons oscillate relativistically, creating gigabar pressure. The displacement of electrons-but not the heavier ions-from the region of the laser focus drives large space-charge fields (exceeding $1 \mathrm{GeV} / \mathrm{cm}$ ). For laser pulses that are short compared with a plasma period, this takes the form of a wakefield, which accelerates MeV energy beams of electrons. For pulses long compared with a plasma period, we show that a Coulomb explosion accelerates protons (or other ions) to energy in excess of $10 \mathrm{MeV}$ in well-collimated beams. In both cases, not only is this acceleration gradient up to a thousand times greater than in radio-frequency accelerators, but we also found that their transverse geometrical emittances are at least comparable, e.g., up to $10^{10}$ particles per pulse and divergence angles as low as 1 for electrons and 20 for protons. Additionally, the repetition rate of the electron gun is $10 \mathrm{~Hz}$, a thousand-fold improvement over its past performance. In order to reduce the large electron energy spread, we show experimentally the injection of electrons into a laser-driven plasma wave by use of a separate synchronized laser pulse. Applications of these subpicosecond duration pulses include laboratory astrophysics, cancer radiotherapy, fast-ignitor fusion, radiochemistry, radiobiology, isotope production, and highcurrent injectors for high-energy and nuclear physics. For instance, with just a single pulse of $\mathrm{MeV}$ deuterons, we created a radioisotope in the reaction ${ }^{10} \mathrm{~B}(\mathrm{~d}, \mathrm{n})^{11} \mathrm{C}$. Similarly, we showed that a single pulse of electrons was sufficient for pulsed radiolysis.
\end{abstract}

\section{INTRODUCTION}

As tabletop lasers continue to reach record levels of peak power, the highest forces ever exerted by light have been used to accelerate beams of electrons and protons to energies of a million volts in distances of only microns. In this paper, we report recent experimental results on a first stage accelerator, in which the electrons or protons are derived from the plasma itself. This might be called a plasma photo-cathode.

We recently reviewed the field of laser-plasma accelerators [1]. In this paper, we concentrate mainly on our own

\footnotetext{
*dpu@umich.edu
}

recent results. First, we present a discussion of the basic physics. Then, we present the results from experiments in which the particles are self-trapped. In Sec. 2.1, we discuss some preliminary experiments in which one laser pulse injects electrons from the plasma into the plasma wave created by another. Lastly, we discuss some prospects for applications.

\section{ELECTRON ACCELERATION}

For time periods that are short compared to an ion period, electrons are displaced from regions of high laser intensity, but ions, due to their much greater inertia, remain stationary. The resulting charge displacement provides an electrostatic restoring force that causes the plasma electrons to oscillate at the plasma frequency $\left(\omega_{p}\right)$ after the laser pulse passes by them, creating alternating regions of net positive and negative charge. The resulting electrostatic wakefield plasma wave propagates at a phase velocity nearly equal to the speed of light and thus can continuously accelerate properly phased electrons.

Acceleration of electrons by electron plasma waves is of current interest because the acceleration gradient (200 $\mathrm{GeV} / \mathrm{m}$ ) is much larger (four orders of magnitude larger) than in conventional $\mathrm{rf}$ linacs $(<20 \mathrm{MeV} / \mathrm{m})[2,3]$. Several methods have been proposed for driving a largeamplitude high-phase-velocity plasma wave, such as the plasma wake-field accelerator, the plasma beat-wave accelerator, the laser wake-field accelerator (LWFA) and the self-modulated laser wake-field accelerator (SMLWFA). The LWFA and the SMLWFA have received considerable attention and shown rapid progress because of the development of table-top ultrashort-duration terawatt-peak-power lasers. In the SMLWFA, an electromagnetic wave $\left(\omega_{o}, \boldsymbol{k}_{\boldsymbol{o}}\right)$ decays into a plasma wave $\left(\omega_{p}, \boldsymbol{k}_{\boldsymbol{p}}\right)$ and another forwardpropagating light wave $\left(\omega_{o}-\omega_{p}, \boldsymbol{k}_{\boldsymbol{o}}-\boldsymbol{k}_{\boldsymbol{p}}\right)$ via the stimulated Raman forward scattering instability. In this case, the laser pulse duration is longer than an electron plasma period, $\tau>>\tau_{p}=2 \pi / \omega_{p}$. We have recently studied theoretically the coherent control of the Raman-driven plasma waves by the use of a frequency chirp on the laser beam. A positive chirp is found to increase the Raman growth rate as compared with no chirp and a negative chirp is found to decrease it [4]. In the LWFA, an electron plasma wave is driven resonantly by a short laser pulse $\left(\tau \sim \tau_{p}\right)$ through the laser ponderomotive force. Self-guiding is possible when laser power exceeds the threshold for relativistic self-guiding, $P_{c}$. Whole beam self-focusing and, more recently, relativistic filamentation (a partial beam analog 
to the whole beam effect, or multiple filaments) have both been observed [5].

Several labs have observed the acceleration of $\mathrm{MeV}$ electrons by the SMLWFA, sometimes accompanied by selfguiding, but with large-electron energy spreads (most of the electrons have energies less than $5 \mathrm{MeV}$, with the number decaying exponentially with a temperature of $\sim 1 \mathrm{MeV}$ to just a few electrons at energies up to $100 \mathrm{MeV}$ ). The origin of the accelerated electrons is a subject of some debate. It has been attributed to catastrophic wave-breaking of a relativistic Raman forward scattered plasma wave, and to wavebreaking of slower velocity Raman backscattered waves in both experiment and theory. A two-temperature distribution in the electron energy spectrum was observed [6] to accompany a multi-component spatial profile of the electron beam. In this case, electrons in the low energy range were observed to undergo an abrupt change in temperature, coinciding with the onset of extension of the laser channel due to self-guiding of the laser pulse, when the laser power or plasma density was varied. With the aid of a test-particle simulation, we have now determined that both of these effects appear to originate from the dynamics of trapping and detrapping as electrons oscillate in their orbits in the seperatrix from regions of acceleration to decelleration and from defocusing to focusing [6].

Laser acceleration of electrons is illustrated in Fig. 1. Here, an intense laser interacts with a gas jet located inside

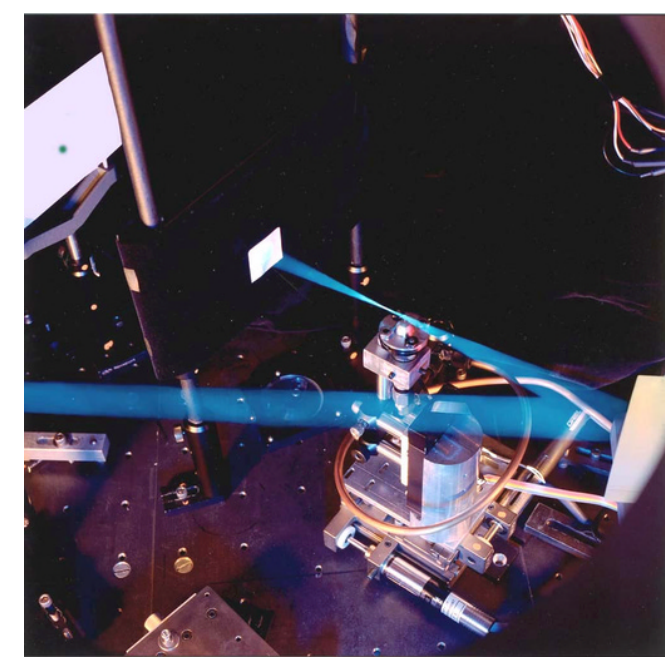

Figure 1: Photograph of the acceleration of an electron beam by a laser interacting with a gas jet inside a vacuum chamber. The laser (illustrated for the purpose of orientation) crosses the picture from left to right and is focused by a parabolic mirror (right side of the picture). The supersonic nozzle (shown in the middle of the picture) is position with micron accuracy with a 3 -axis micropositioner. The e-beam makes a small spot on a white florescent (LANEX) screen, shown in the upper left-hand corner of the picture.

a vacuum chamber. The laser crosses the picture from left to right and is focussed by a parabolic mirror (right side of the picture). The supersonic nozzle (shown in the middle of the picture) is positioned with micron accuracy with a 3axis micropositioner. The e-beam (up to $10^{10}$ electrons per shot) makes a small spot on a fluorescent (LANEX) screen (imaged with a CCD camera), shown in the upper left-hand corner of the picture. As shown in Fig. 1 [6], as the laser power increases, the divergence angle of the electron beam decreases. The lowest angle, $1^{\circ}$, obtained at the highest power, corresponds to a transverse geometrical emittance of $\epsilon_{\perp} \leq 0.06 \pi$ mm-mrad [6], which is an order of magnitude lower than that from the best conventional electron gun. This may be because a large acceleration gradient decreases the time over which space-charge can act to degrade the emittance.

\subsection{Optical Injection}

The injection of electrons into plasma waves can occur uncontrollably by trapping of hot background electrons, which are preheated by other processes such as Raman backscattering and sidescattering instabilities [7], or by wave-breaking (longitudinal [8] or transverse [9]). Because the electrons in this case are injected into the plasma wave uniformly in phase space, large energy spreads result, as is typically observed in the SMLWFA regime. The injection can also be controlled by use of an external electron source (such as from an RF gun); however, because the pulse durations of the injected electron bunches in the experiments in which this method was tried were longer than the acceleration buckets, the energy spread was again large. It has been shown analytically and numerically that controlled injection might also be accomplished by means internal electrons, from the plasma itself, which are all put into the accelerating phase of the plasma wave by a separate laser pulse $[10,1]$. Such a laser-driven plasma-cathode electron gun might eventually have (1) monoenergetic energy, (2) $\mathrm{GeV} / \mathrm{cm}$ acceleration fields, (3) micron source size, (4) femtosecond pulse duration, (5) high brightness, (6) absolute synchronization between electrons and laser (for pump and probe experiments) and (7) compact size (university-lab scale).

As a first step towards the realization of monoenergetic beams by means of optical injection, we have studied experimentally, in the SMLWFA regime, a concept first discussed in the LWFA regime [10]. By crossing in a plasma two beams, each of duration $400 \mathrm{fs}$ and wavelength $1 \mu \mathrm{m}$, one with high vacuum intensity $\left(a_{0}=1.0\right)$ and the other lower intensity $\left(a_{0}=0.25\right)$, we find that only when the beams are overlapped in space and time do we observe what appears to be diffraction of light from a standing wave (see Fig. 3) and the acceleration of electrons in the direction of the low power beam (see Fig. 4). Injection in this case is caused by the ponderomotive force of a standing wave created along the bisector of the two beams, which will stochastically heat the electrons to relativistic temperature and allow them to be caught in the plasma wave that was created by the low power beam. This process is similar to the "preheating" that occurs when the slow-phase- 


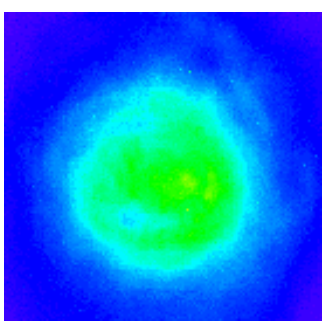

(a)

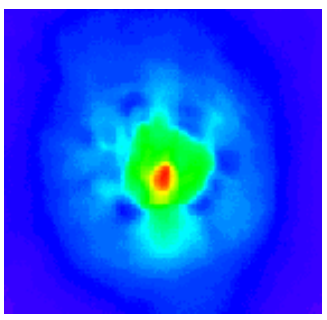

(c)

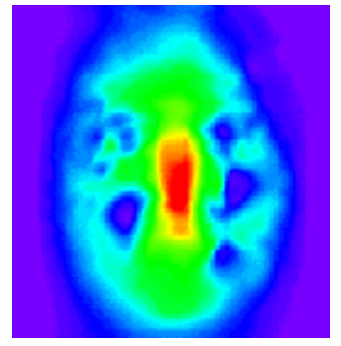

(b)

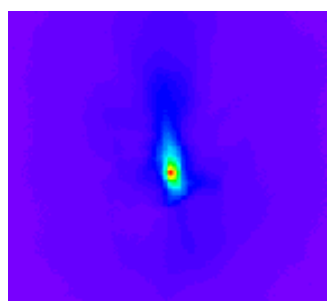

(d)

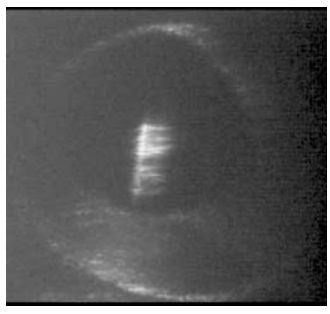

(a)

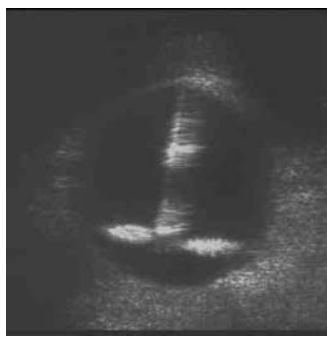

(c)

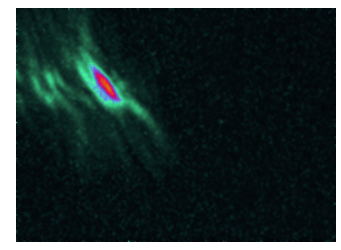

(b)

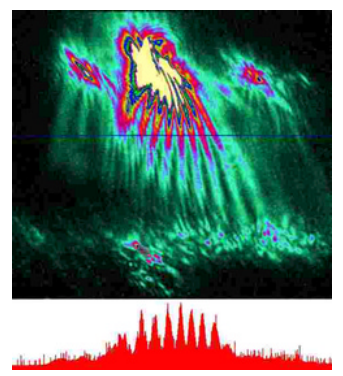

(d)
Figure 2: Images of the spatial profiles of the electron beam measured by a ccd camera imaging a LANEX screen at a distance of $15 \mathrm{~cm}$ from the gas jet for various laser powers: a) $0.6 \mathrm{TW}, \mathrm{b}) 1.1 \mathrm{TW}, \mathrm{c}) 2.0 \mathrm{TW}$, d) $2.9 \mathrm{TW}$. The divergence angle of the beam for (d) decreases to a value of $\Delta \theta=1^{\circ}$ at a power of $2.9 \mathrm{TW}$, corresponding to a transverse geomtrical emittance of just $\epsilon_{\perp} \preceq 0.06 \pi$ mm-mrad.

velocity plasma wave driven by Raman backscattering injects electrons into the faster plasma waves that are generated by Raman forward scattering [7]. However, in this case, the ponderomotive force of the standing wave is much higher because of its much shorter wavelength. It is also occasionally observed that the increase of the index of refraction resulting from the addition of the pulses can extend the distance over which the low-power beam remains focused. This is the first time that one laser beam has been used to trigger the acceleration of electrons by another laser beam, thus proving the LILAC principle.

\section{PROTON ACCELERATION}

Plasma ions can be accelerated to high energies by the formation of an electrostatic sheath due to charge displacement. The latter results from the initial preferential acceleration of electrons; the heavier ions are left behind due to inertia. Ions will also be accelerated by each other's unshielded charges in what has been termed a "Coulomb explosion." Among the many mechanisms that can accelerate the electrons are: thermal expansion, plasma waves, " $J \times B$ heating" or "vacuum heating." Recent simulations reveal a new mechanism [11] that occurs in cases where a significant preplasma exists, such as for pulses with typical laserintensity contrast ratios. In this case, a standing wave is produced by the beating of the incident and reflected light,
Figure 3: (a, c) Images of the Thomson scattered light as viewed from the top looking down the throat of the nozzle: (a) single pulse, (c) two crossed pulses. (b, d) Images of the scattered light as viewed from the side along the bisector; (b) single pulse, (d) two crossed pulses. The latter (d) appears to shows a diffraction pattern characteristic of Bragg scattering from a grating (periodically bunched electrons), presumed to result from the ponderomotive force of the standing wave created by the beating of the two light waves.

which can heat the electrons to relativistic temperatures, as was described in the discussion of optical injection (Sec. 2.1).

\subsection{Experiments}

Energetic ions from underdense plasmas were accelerated by an electrostatic sheath, which was created by charge-displacement. Unlike earlier long-laser-pulse experiments, the displacement was due not to thermal expansion but to ponderomotive blow-out $[12,13]$. When a helium-gas was used as the target, alpha particles were accelerated to several $\mathrm{MeV}$ in the direction orthogonal to the direction of laser propagation and along the direction of the maximum intensity gradient.

Several groups have reported the observation of ions originating from thin-film solid-density targets (or protons originating from monolayers of water on the target surface). Unlike previous long-pulse experiments, the protons were accelerated along the direction normal to the side of the target opposite to that upon which the laser was incident. For instance, Fig. 5 shows schematically the typical set-up. Here, the laser (shown in the foreground) is focused with an off-axis parabola onto a thin-foil, held by 


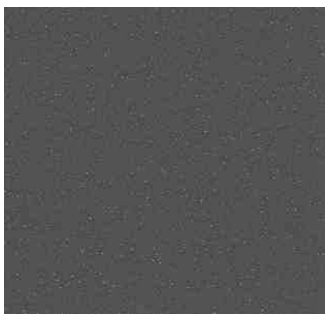

(a)

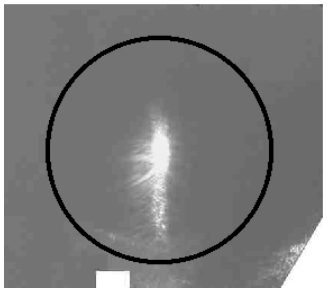

(c)

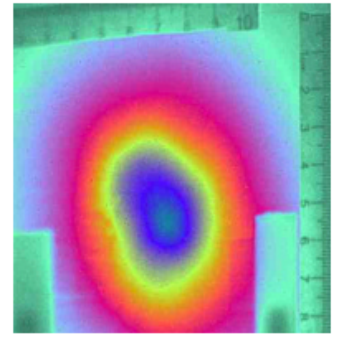

(b)

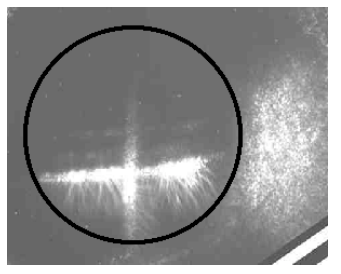

(d)
Figure 4: $(a, b)$ Images of the LANEX screen in the direction of the low-intensity beam; (a) low-intensity pulse alone, (b) two crossed pulses. (c, d) Images of the Thomson scattered light as viewed from the top looking down the throat of the nozzle; (c) low-intensity pulse alone, The black circles are added to show the boundaries of the inner diameter of the nozzle orifice. (d) two crossed pulses. Note that an electron beam is only observed (b) in the case of two overlapping beams (d).

a mesh that is positioned by a 3 -axis micropositioner. A nuclear track detector, CR-39 (shown in white in the background), shows an actual proton-produced pattern [14]. In another experiment, protons were observed to be emitted in ring patterns, the radii of which depend on the proton energy, which was explained by self-generated magnetic fields [15]. Another recent result reported proton energies up to $60 \mathrm{MeV}$ [16]. The results of these experiments indicate that a large number of protons $\left(10^{13} p\right)$ can be accelerated, corresponding to current densities $\left(10^{8} \mathrm{~A} / \mathrm{cm}^{2}\right)$ at the source that are nine orders-of-magnitude higher than produced by cyclotrons, but with comparable transverse emittances $\left(\epsilon_{\perp} \leq 1.0 \pi \mathrm{mm}\right.$-mrad). The high end of the energy spectrum typically has a sharp cut-off, but, like the electrons is a continuum.

While the protons in several experiments originate from the front-side of the target $[14,15]$, in another [16], they originate from the back-side. Evidence for a back-side origin comes from results obtained when wedge-shaped targets were used. The proton beam was observed to point in the direction normal to the back side of the target, which was not perpendicular to the front surface. On the other hand, evidence for a front-side origin comes from an experiment in which deuterium was coated on a thin film of mylar and a boron target was placed behind it [17]. It was found that only when the deuterium was on the

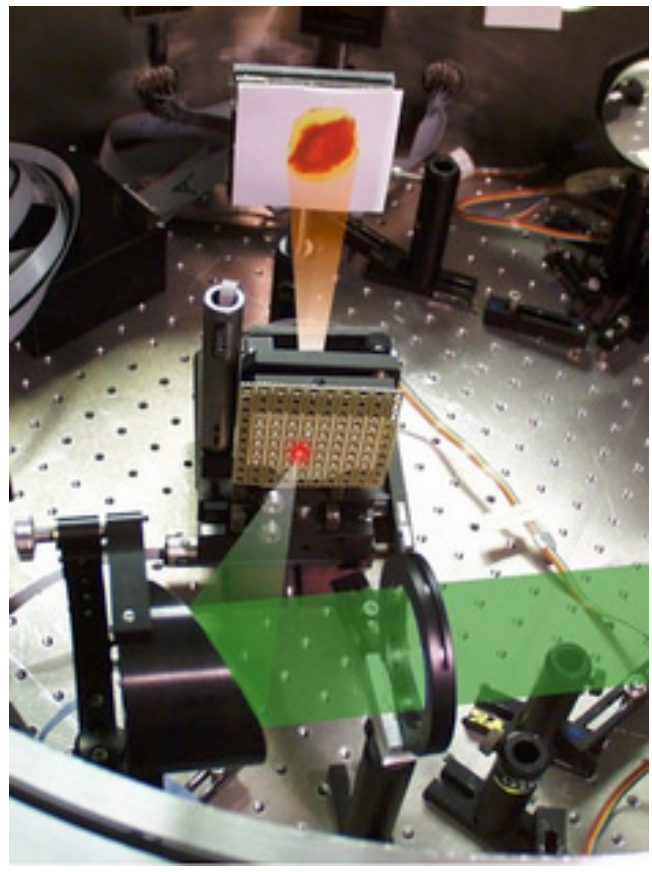

Figure 5: Photograph of the typical setup used to observe the acceleration of ions. The laser beam (illustrated in the foreground) is focused with an off-axis parabola onto a thin-foil, held by a mesh that is positioned by a 3-axis micropositioner. CR-39, a nuclear track detector (shown in white in the background) is used to detect the ions. An actual proton-produced pattern is shown.

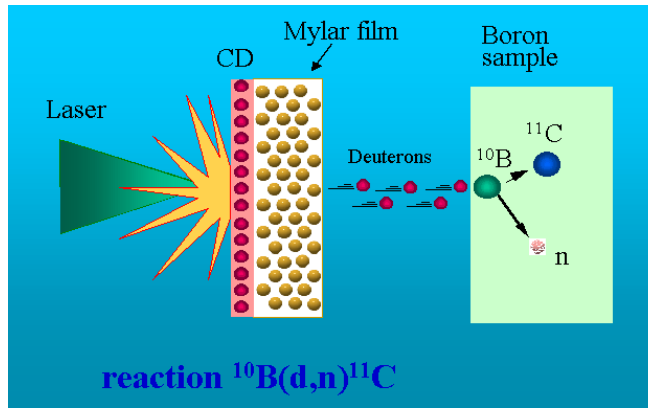

Figure 6: Schematic of the experimental set-up used to create a radioisotope with laser-accelerated deuterons.

front side did the boron become activated by the reaction ${ }^{10} \mathrm{~B}(d, n){ }^{11} \mathrm{C}$.

\section{PROSPECTS}

A promising avenue for the production of $\mathrm{x}$-rays is based on the use of Compton scattering. Upon scattering with an electron beam, laser light can be upshifted due to a relativistic boost by a factor of $4 \gamma^{2}$, which for electrons accelerated to $30 \mathrm{MeV}$ corresponds to a factor of 10,000. Thus a $1 \mathrm{eV}$ photon can be upshifted to $10 \mathrm{keV}$. Such coherent, ultrashort duration and energetic sources could enable ultrafast imaging on the atomic scale. If the source of the 


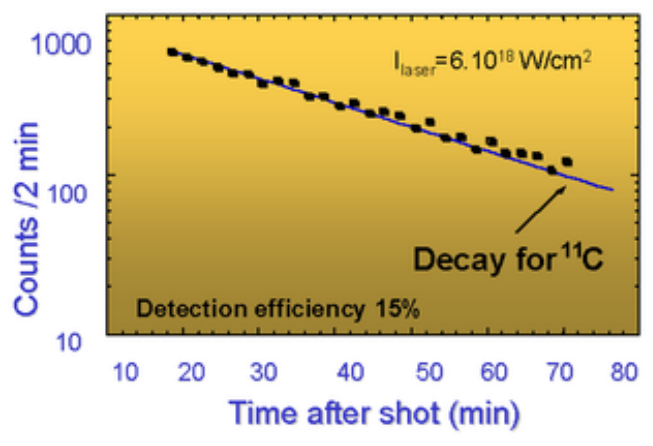

Figure 7: Experimentally recorded radioactive decay rate corresponds to that of ${ }^{11} \mathrm{C}$, which decays by positron emission. The $511 \mathrm{keV}$ gamma rays which were detected were created by annihilation of the electron-positron pairs.

electron beam were a laser accelerator, the footprint of this synchrotron-like device would be small enough for it to fit in a university laboratory.

It was demonstrated that there are a sufficient number of electrons accelerated by laser-plasma accelerators to conduct pulsed radiolysis [18]. Time-resolved radiobiological studies with laser accelerated protons are also feasible.

It has been shown theoretically that a laser-induced burst of hot electrons or ions could be used as a spark to ignite a thermonuclear reaction with inertial confinement fusion in the so-called fast-ignitor laser fusion concept. In its original conception, a short but energetic laser pulse would drill through the under-dense plasma that surrounds the fusion core and a second shorter pulse would deposit energy in the core in the form of $\mathrm{MeV}$ electrons. In so doing, it would relax the otherwise stringent requirements on energy and symmetry of the long-pulse-duration heating and compression pulses. More recently, the use of a short pulse of ions for the ignitor has also been discussed.

The short-lived radioactive isotopes that were produced by laser acceleration of ions might be used to for cancer diagnostics such as in positron-emission tomography. Because they are created impulsively with a ion short pulse, they might also be used to study ultrashort duration isotope decay times in nuclear physics. If protons could be laser-accelerated to $70-160 \mathrm{MeV}$ energies, they could be useful for proton therapy, which is now limited by the extraordinary expense of cyclotrons or synchrotrons and the large magnets required to transport the proton beams to the patient. Protons are superior to other forms of ionizing radiation for cancer treatment because of less straggling and their ability to deposit their energy over a narrower depth range.

The authors would like to acknowledge the support of the High Energy Physics Division, U.S. DOE, award DEFG02-98ER41071 (electrons); Chemical Sciences, Geosciences and Biosciences Division, U.S. DOE (ions and $\mathrm{x}$ rays); NSF (laser facilities and plasma physics).

\section{REFERENCES}

[1] D. Umstadter, Phys. Plasmas 8, 1774 (2001), and references therein.

[2] T. Tajima and J. M. Dawson, Phys. Rev. Lett. 43, 267 (1979).

[3] E. Esarey, P. Sprangle, J. Krall, and A. Ting, IEEE Trans. Plasma Sci. PS-24, 252 (1996), and references therein.

[4] E. Dodd and D. Umstadter, Phys. Plasmas, (to appear, 2001).

[5] X. Wang, M. Krishnan, N. Saleh, H. Wang and D. Umstadter, Phys. Rev. Lett. 84, 5324 (2000).

[6] S.-Y. Chen, M. Krishnan, A. Maksimchuk, R. Wagner and D. Umstadter, Phys. of Plasmas 6, 4739 (1999).

[7] P. Bertrand, A. Ghizzo, S. J. Karttunen, T. J. H. Pattikangas, R. R. E. Salomaa, and M. Shoucri, Phys. Rev. E 49, 5656 (1994).

[8] A. Modena, Z. Najmudin, A. E. Dangor, C. E. Clayton, K. A. Marsh, C. Joshi, V. Malka, C. B. Darrow, C. Danson, D. Neely, and F. N. Walsh, Nature 377, 606 (1995).

[9] S. V. Bulanov, F. Pegoraro, A. M. Pukhov, and A. S. Sakharov, Phys. Rev. Lett. 78, 4205 (1997).

[10] D. Umstadter, J. K. Kim, and E. Dodd, Phys. Rev. Lett. 76, 2073 (1996).

[11] Y. Sentoku et al., Phys. Plasmas (submitted, 2001).

[12] G. S. Sarkisov, V. Yu. Bychenkov, V. N. Novikov, V. T. Tikhonchuk, A. Maksimchuk, S. Y. Chen, R. Wagner, G. Mourou and D. Umstadter, Phys. Rev. E 597042 (1999).

[13] K. Krushelnick, E. L. Clark, Z. Najmudin, et al., Phys. Rev. Lett. 83, 737 (1999).

[14] A. Maksimchuk, S. Gu, K. Flippo, D. Umstadter, V.Y Bychenkov, Phys. Rev. Lett. 84, 4108 (2000).

[15] E. L. Clark K. Krushelnick, J. R. Davies, et al., Phys. Rev. Lett. 84, 670 (2000).

[16] R. A. Snavely, M. H. Key, S. P. Hatchett, et al., Phys. Rev. Lett. 85, 2945 (2000).

[17] K. Nemoto, A. Maksimchuk, S. Banerjee, K. Flippo, G. Mourou, D. Umstadter, V. Yu. Bychenkov, Appl. Phys. Lett. 78, 595 (2001).

[18] N. Saleh, K. Flippo, K. Nemoto, D. Umstadter, R. A Crowell, C. D Jonah, A. D Trifunac, Rev. Sci. Instr., 71, 2305 (2000). 